\title{
Commentary: The mechanics of acute aortic dissection: Measured calculations and calculated measures
}

\author{
Keshava Rajagopal, MD, PhD, ${ }^{\mathrm{a}}$ Boyce E. Griffith, $\mathrm{PhD},{ }^{\mathrm{b}}$ and Abe DeAnda, Jr, $\mathrm{MD}^{\mathrm{c}}$
}

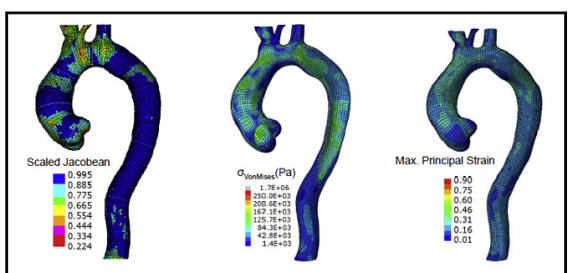

Patient-specific aortic dissection modeling.

Central Message

A patient-centric predictive model for the development of an aortic dissection has promise for improving our understanding of the disease process and potentially being used as a clinical tool.

See Article page 355 .
I often say that when you can measure what you are speaking about, and can express it in numbers, you know something about it; but when you cannot measure it, when you cannot express it in numbers, your knowledge is of a meagre and unsatisfactory kind; it may be the beginning of knowledge, but you have scarcely, in your thoughts, advanced to the stage of science, whatever the matter may be.

— William Thomson 1st Baron Kelvin

Electrical Units of Measurement

In this issue of the Journal, Emerel and colleagues ${ }^{1}$ report a novel study pertinent to the mechanics underlying acute thoracic aortic dissection (TAD). The central finding of this work is that local axial normal stresses may be more important to the pathogenesis of TAD than circumferential normal stresses, a finding that is distinct from the extant body of literature.

In a cohort of patients who eventually developed acute TAD, a subset happened to undergo transthoracic echocardiography (and computed tomography [CT] angiography) and simultaneous blood pressure assessment, yielding measurements of (1) aortic motion during the cardiac cycle and (2) peripheral arterial pressure, the latter via noninvasive means and in an extremity. From these measurements, aortic material properties were calculated. By using these data, overlaid onto aortic geometry obtained from CT angiography, local aortic wall stresses were calculated-both axial and circumferential (radial stresses are typically much lower)-and compared with data from a control group of patients with presumably normal aortas who did not experience TAD. In addition, intraoperatively identified entry tear sites were overlaid onto the derived stress maps. The authors found that (1) ascending thoracic aortic distensibility was lower and stiffness higher in aortas that eventually experienced TAD; (2) derived axial normal stresses, but not circumferential normal stresses, were higher in aortas that eventually experienced TAD; and (3) entry tears were found to occur in areas of high calculated axial normal stresses.

A general principle of mechanics, first expressed in Newton's Second Law, takes the form of force (or some similar dynamical vector concept such as stress) equals some scalar parameter of material inertial properties (ie, an expression of the tendency to impede or resist motion) multiplied by some vector kinematic output (eg, acceleration in Newton's second law or strain in stress-strain relationships). Such equations, although expressing valid relationships, do not convey the causality of the relationships. It was Newton's genius that he understood that force causes motion. Likewise, the authors appreciate that aortic stresses and aortic material properties are the independent and causative variable of interest with respect to the dependent variable of aortic motion, and they specifically show that calculated axial, but not circumferential stresses, are abnormally elevated.

There are limitations in the modeling approach used in this study. First, the aorta is assumed to be an isotropic, homogeneous material without layers. That is, although stresses are permitted to vary spatiotemporally, as encoded by CT scan-obtained aortic geometry, aortic material parameters are not. Yet the aorta is an anisotropic, inhomogeneous, layered composite. Because TAD is an interplay between local aortic forces and local aortic material properties, is it reasonable to assume spatiotemporal variations in one independent variable but not the other? 
Moreover, because the very definition of TAD is aortic wall delamination, it seems questionable to assume the absence of the structure that defines the process of TAD. Second, although most groups that have studied TAD and aneurysmal disease have focused on normal stresses, or stresses perpendicular to/through surfaces, TAD itself-or at least its propagation-critically involves shear stresses, the stresses that act parallel to/along surfaces. The actual dissection process of blood flow delaminating the aorta is one of shear-induced injury. Third, although the authors do attempt to discern aortic material properties, they do so by determining parameters from limited measurements. This gets to the heart of Kelvin's famous quotation regarding measurement. Aortic distensibility and stiffness are ascertained using blood pressure measurements. However, the idea of a pressure that is spatially invariant through the systemic arterial circulation (peripheral arterial pressure is assumed identical to central aortic pressure) is unsound and is linked to assumptions of arterial homogeneity. Thus, we are left with a bit of a puzzle: TAD may arise in the setting of abnormal aortic loading conditions and abnormal aortic properties, abnormal aortic loading conditions and normal aortic properties, and normal aortic loading conditions and abnormal aortic properties.
Yet, because aortic properties are obtained via idealized approaches, the validity of the model chosen to capture aortic mechanical properties is unclear. Although the patients studied appear to be in the first group of conditions, they might actually be in the second or even third groups. This has obvious practical implications with respect to medical therapies and if such studies are used to predict TAD risk and impending surgical treatment.

Despite these limitations, this study is an important contribution to our understanding of TAD. All models have inherent simplifications, and in some cases, simple models may be improved by adding additional complexities. However, many contemporary studies of aortic mechanics are limited by unreasonable simplifying assumptions. It seems warranted to verify the predictions against more sophisticated mathematical models with more directly measured parameters to determine the optimal balance in model complexity.

\section{Reference}

1. Emerel L, Thunes J, Kickliter T, Billaud M, Phillippi JA, Vorp DA, et al Predissection-derived geometric and distensibility indices reveal increased peak longitudinal stress and stiffness in patients sustaining acute type A aortic dissection: implications for predicting dissection. J Thorac Cardiovasc Surg. 2019; 158:355-63. 